\title{
DOES THE USE OF THE OPERATING MICROSCOPE IMPROVE THE RESULTS OF PERIPHERAL NERVE SUTURE?
}

\author{
DAVID MARSH, NICHOLAS BARTON
}

\author{
From the Harlow Wood Orthopaedic Hospital, Nr Mansfield
}

\begin{abstract}
Twenty-four cases of complete division of median or ulnar nerves were assessed on two occasions after direct suture, with an interval of three years between assessments. Clinical, electrophysiological and timed functional tests were used. All the operations had been performed by one surgeon, using the operating microscope for approximately half the cases but not for the other half.

The results were analysed, and the patient's age, any delay between injury and suture, and the duration of follow-up were all found to exert strong and consistent effects on the scores obtained. After controlling for these factors, there was no consistent difference between the results of the two surgical methods. It is concluded that, at least in the hands of one particular surgeon, the use of the operating microscope gives no better results than careful epineural suture performed without it.
\end{abstract}

It has been the hope of many surgeons who treat peripheral nerve injuries that microsurgical techniques might lead to a better functional result, due to gentler handling of neural tissue, the use of finer suture material and more accurate coaptation of fasciculi. Moreover, the operating microscope makes it possible to place sutures accurately in the perineurium surrounding individual fascicles.

The difficulties of making objective assessments of nerve function prevent comparison between results from different centres at present. Therefore the demonstration of such benefit requires comparative studies, in which patients whose nerves have been sutured by different techniques are examined by identical methods. So far there has been a remarkable dearth of such studies in humans, and none has yet convincingly shown advantage from the use of the operating microscope.

Salvi (1973) published a personal series containing the results of six epineural and 16 perineural interfascicular sutures of recently divided nerves, together with some interfascicular grafts and late epineural repairs. He reported better results in the perineural cases, with an earlier reappearance of sensibility. However, his results are difficult to interpret because the series comprised a mixture of digital and mixed nerves and of

D. Marsh, FRCS, Lecturer in Orthopaedics, Manchester University Clinical Sciences Building, Hope Hospital, Eccles Old Road, Salford M6 8HD, England.

N. J. Barton, FRCS, Consultant Orthopaedic and Hand Surgeon Harlow Wood Orthopaedic Hospital, Nr Mansfield, Nottingham NG18 4TH, England.

Requests for reprints should be sent to Mr D. Marsh. (C) 1987 British Editorial Society of Bone and Joint Surgery $0301-620 \mathrm{X} / 87 / 4124 \$ 2.00$ direct sutures and grafts. In addition, there appears to have been a preponderance of young patients among the good perineural cases. Finally, the role of the operating microscope itself cannot be assessed since all the earlier operations, including some of the fascicular repairs, were performed without it (Salvi, personal communication).

Donoso, Ballantyne and Hansen (1979) reported a series of 67 ulnar or median nerve sutures, six of which were epi-perineural, performed by various surgeons. The epi-perineural cases showed a faster returned of motor and sensory action potentials, but again the method was selectively used in younger patients.

Young, Wray and Weeks (1981), in a randomised prospective trial of epineural versus perineural suture of digital nerves, showed no difference between the two techniques.

Some comparative studies in non-human animals have shown apparent enhancement of reinnervation after perineural, as opposed to epineural, suture. Others have shown no difference. However, the relevance of these studies is questionable, partly because the vigour of regeneration is much higher in such species and partly because the neural basis for good integrated hand function after suture is not known.

Thus it can be said that there is as yet no firm evidence to suggest that a patients with a divided median nerve will regain better hand function if the nerve is sutured by a microsurgical technique. Those few series which have suggested benefit are inconclusive, either because they contain a mixture of lesions and treatments or because potent factors such as the age of the patient have not been tightly enough controlled in analysing the results. The present study constitutes an attempt to overcome these problems. 


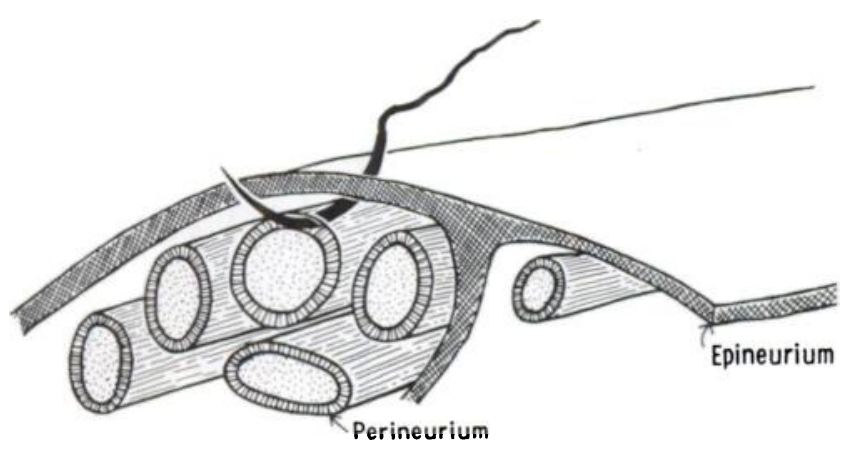

Fig. 1

Diagram of epi-perineural suture placement. The suture includes the perineurium of the outer fascicles only. (Reproduced, with permission, from the British Journal of Occupational Therapy 1986;49:79.)

\section{PATIENTS}

Two groups of patients were assessed after nerve suture. One group had epi-perineural repairs (Fig. 1) performed under the operating microscope, with no interfascicular dissection or excision of epineurium. The other cases had epineural repairs, performed either without magnification or with the aid of loupes.

The series was selected first and foremost for its homogeneity. All cases were complete divisions of median or ulnar nerves between elbow and wrist; there were no untidy or contaminated wounds. All were treated by direct suture between 1969 and 1981 in Nottingham. Every operation, with or without the microscope, was wholly performed by the same surgeon (NJB).
Every available patient who met all these criteria was assessed, but one patient, with two divided nerves, was eventually excluded. He was 70 years old, and had cut his own wrist in a suicide attempt. When seen two years after operation, he had a completely frozen hand, and this prevented any functional assessment of neurological recovery.

The application of these strict criteria (particularly the exclusion of incomplete cuts and operations performed partly or wholly by other surgeons) led to a relatively small series of 21 patients with 24 divided nerves: 11 in the microscope group, 13 in the epineural group. This was felt to be a correct design decision, since broadening the net to gain more cases would have introduced other factors which would have rendered valid comparison impossible.

Of the 24 nerve sutures, 13 were median and 11 ulnar. These were evenly distributed between the two groups (Table I). The age distribution slightly favoured the no-microscope group (Fig. 2), their mean age being 19.4 years compared to 24.6 years in the microscope group.

The delay between injury and suture also differed, this time favouring the microscope group (Fig. 3), several patients in the no-microscope group being referred to us long after injury. The difference is considerable, with a mean delay between injury and operation of 12.5 days in the microscope group, and 49.7 days in the no-microscope group.

However, the biggest disparity between the groups was in the time between operation and assessment (Fig. 4). This stemmed simply from the fact that the

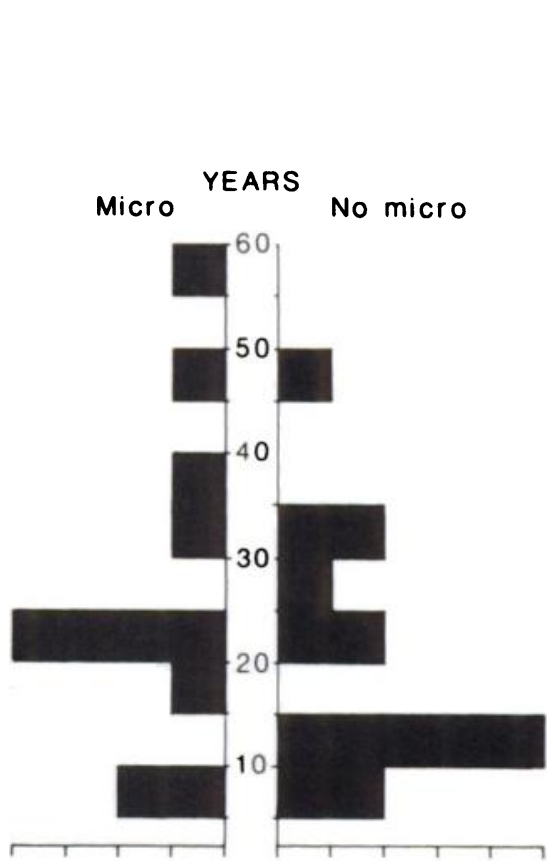

Fig. 2

Age distribution at the time of suture.

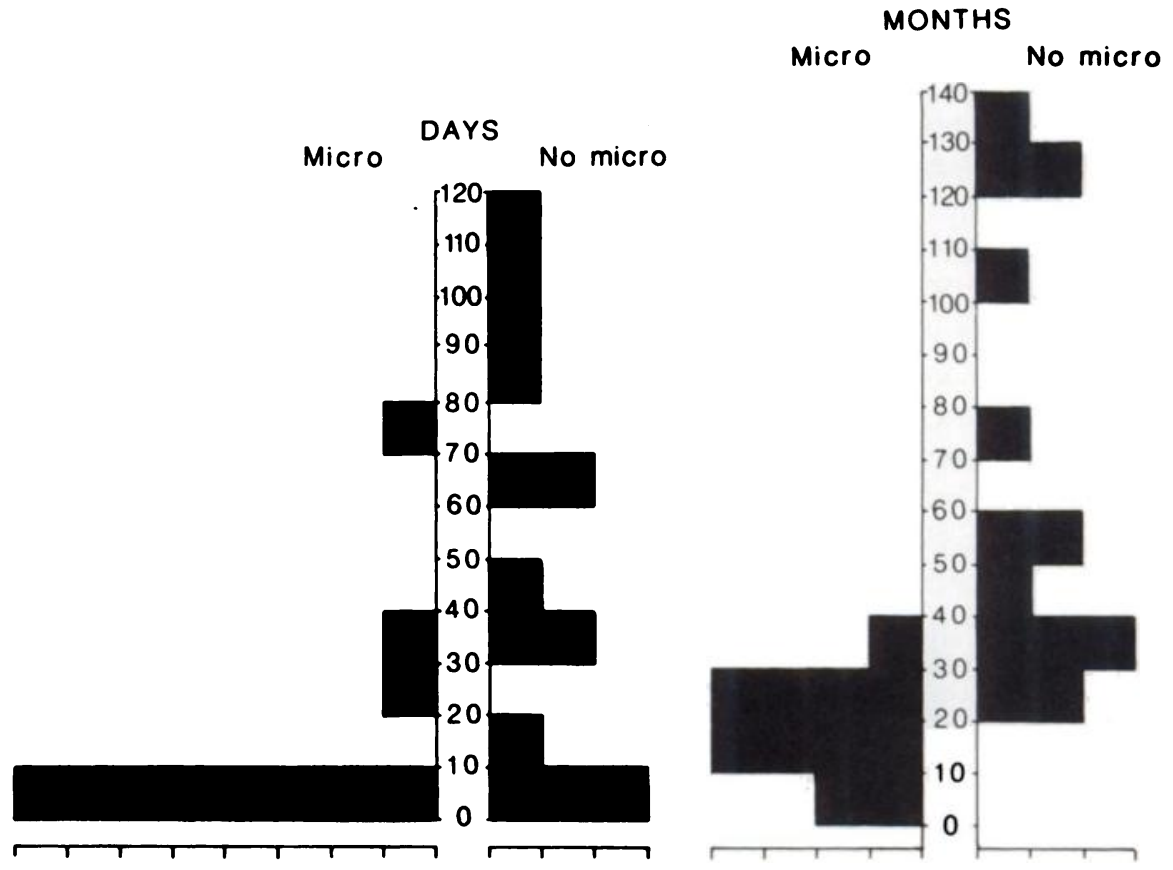

Fig. 3

Delay between injury and suture.
Fig. 4

Time elapsed between suture and the first examinations in 1982. 
microscope was purchased in 1979 and was used for most cases thereafter. The first wave of examinations was carried out in 1982, when the average follow-up time was 17.6 months in the microscope group and 66.2 months in the no-microscope group.

Table I. Distribution of nerves between the groups

\begin{tabular}{lll}
\hline & Microscope & No microscope \\
\hline Median & 6 & 7 \\
Ulnar & 5 & 6 \\
\hline
\end{tabular}

\section{METHODS}

All patients were examined in 1982. Sixteen of them, with 18 injuried nerves (nine from each group), were reexamined by the same personnel, using the same methods, in 1985. In all tests, performance in the injured hand was compared with that of the normal hand. In this way much of the individual variation in performance (due to age, intelligence, skin thickness and so on) is controlled. All results are expressed as a percentage of the normal side, calculated so that a higher score means a better function. The battery of tests used falls into three categories.

Specific motor and sensory tests. Muscle power was scored on the MRC 0 to 5 scale by comparison with the normal hand. For median nerve lesions, the power of abduction and opposition of the thumb were assessed. For ulnar nerve lesions the power of the first dorsal interosseous, abductor digiti minimi and adductor pollicis muscles were assessed.

Spatial discrimination was tested in two ways. First, the moving two-point discrimination (2PD) test was performed (by DM) in the manner described by Dellon (1978), using a paper-clip. Threshold was taken as the smallest separation of the points which could be correctly distinguished seven times out of 10 , applications of one or two points being made in random order. Second, two new wheel aesthesiometers were employed, using the method described by Marsh (1986).

Localisation was tested by an occupational therapist who was unaware of the group to which the patient belonged. The hand was hidden from the patient's sight by an oblique wooden screen. The territory of the injured nerve was divided into zones of approximately the size of one phalanx: 16 for the median nerve, nine for the ulnar. Patients were tested on their ability to localise correctly a light stroking touch in each zone. Correct localisation scored two points, an immediately adjacent zone scored one, anything worse scored zero.

In addition to the above quantitative tests, protective sensation was assessed by the ability to distinguish reliably between the sharp and blunt ends of a hat-pin. All patients could do this.
Electrophysiological tests. Conventional nerve conduction studies were performed by a consultant clinical neurophysiologist (who was also unaware of whether the microscope had or had not been used), after the hand had been warmed to $32^{\circ} \mathrm{C}$ if necessary.

The fastest motor conduction velocity was measured from elbow to wrist, the shortest distal motor latency from wrist to the relevant intrinsic muscle (Hodes, Larrabee and German 1948). In most cases, therefore, velocity was measured above the lesion and latency across and below it. The supramaximally evoked muscle action potential was recorded through surface electrodes and the peak motor amplitude measured.

The relevant digital nerves were stimulated through ring electrodes and sensory action potentials (SAP) recorded at the wrist. On only five occasions (out of 42) could no SAP be observed. Sensory latency (both to onset and to peak of the SAP), maximum sensory conduction velocity and peak sensory amplitude were measured. A simple measure of the degree of temporal dispersion of the SAP was derived by subtracting latency to onset from latency to peak. An estimate of cumulative sensory amplitude was attempted to calculating the area under the SAP as (approximately) dispersion multiplied by peak amplitude.

Tests of integrated function. Timed functional tests were carried out by an occupational therapist. These were designed on the lines advocated by Moberg (1958) and Wynn-Parry, Sutcliffe and Millar (1966) and involved identification of shapes and textures and picking-up of small objects. Details of the methods have been given elsewhere (Marsh and Smith 1986).

All the data were held on the Cambridge University Data Network and analysed on an IBM 3081, using the Statistical Package for the Social Sciences (SPSS-X).

\section{RESULTS}

Table II shows the scores for the two groups in 1982. Since the wheel aesthesiometers tended to replicate the moving $2 \mathrm{PD}$ results, they are not reported here. At that time, all tests showed the microscope group to be inferior to the no-microscope group. However, as already mentioned, there were big differences in follow-up times.

Figure 5 indicates that recovery continues at a significant rate for about four years from suture. Since the two treatment groups were concentrated on different parts of the fitted curve (which was of similar shape for nearly all tests), it was not possible to make definitive comparisons between them in 1982. The study was therefore suspended for three years, with the prediction that results in 1985 should represent the plateau phase for all patients.

Table II also summarises the results in 1985 for each test. The microscope group more than caught up on muscle power and moving 2PD, but did not do so on the localisation score. The functional tests also showed much 
more improvement among the microscope cases, particularly the pick-up test. The motor amplitude showed marked improvement, but there was some further improvement in the epineural group, indicating perhaps that maturation of motor units continues for even longer. However, the amplitude of the sensory action potential, which is thought to represent roughly the number of conducting fibres in the nerve, showed that, while the nomicroscope group remained static, the microscope group failed to catch up. The fastest motor and sensory velocities, which tend to recover more quickly, did not show a rise over the three years. However, the SAP dispersion in the microscope group did improve, suggesting that there were changes in the distribution of conduction velocities. amplitude, velocity and dispersion of the sensory action potential. Figure 6 shows the two-point discrimination results as a function of age. This shape of curve provided the best fit for the sensibility tests, the pick-up test and almost all the electrophysiological measures. However, muscle power and the other functional tests tended to show a more linear relationship.

Interestingly, delay between injury and suture had much stronger effects on the functional scores than on the electrophysiological measures. Possibly this reflects adaptation by the patient, during the period of denervation, shifting preference to the uninjured hand. Delay also led to worse recovery of muscle power, although there was no discernible effect on sensibility. Figure 7 depicts the effect of delay on a combined score of all the

Table II. Mean test scores for the two groups in 1982 and 1985

\begin{tabular}{|c|c|c|c|c|c|}
\hline & \multicolumn{3}{|c|}{ Mean scores in 1982} & \multicolumn{2}{|c|}{ Mean scores in 1985} \\
\hline & $\begin{array}{l}\text { Mieroscope } \\
(n=11)\end{array}$ & $\begin{array}{l}\text { No microscope } \\
(n=13)\end{array}$ & $\mathbf{p}$ & $\begin{array}{l}\text { Mieroscope } \\
(n=9)\end{array}$ & $\begin{array}{l}\text { No microecope } \\
(n=9)\end{array}$ \\
\hline $\begin{array}{l}\text { Muscle power } \\
\text { Moving 2PD } \\
\text { Localisation }\end{array}$ & $\begin{array}{r}55 \\
9 \\
83\end{array}$ & $\begin{array}{l}69 \\
15 \\
95\end{array}$ & $\begin{array}{l}0.20 \\
0.29 \\
0.11\end{array}$ & $\begin{array}{l}73 \\
20 \\
88\end{array}$ & $\begin{array}{l}66 \\
19 \\
99\end{array}$ \\
\hline $\begin{array}{l}\text { Shapes } \\
\text { Textures } \\
\text { Pick up }\end{array}$ & $\begin{array}{l}32 \\
36 \\
54\end{array}$ & $\begin{array}{l}48 \\
55 \\
66\end{array}$ & $\begin{array}{l}0.02 \\
0.07 \\
0.31\end{array}$ & $\begin{array}{l}50 \\
40 \\
77\end{array}$ & $\begin{array}{l}43 \\
47 \\
62\end{array}$ \\
\hline $\begin{array}{l}\text { Motor amplitude } \\
\text { Motor velocity } \\
\text { Motor latency }\end{array}$ & $\begin{array}{l}33 \\
99 \\
72\end{array}$ & $\begin{array}{l}58 \\
92 \\
84\end{array}$ & $\begin{array}{l}0.01 \\
0.39 \\
0.07\end{array}$ & $\begin{array}{l}57 \\
97 \\
75\end{array}$ & $\begin{array}{l}67 \\
86 \\
81\end{array}$ \\
\hline $\begin{array}{l}\text { SAP peak } \\
\text { SAP cumulative } \\
\text { SAP velocity } \\
\text { SAP dispersion }\end{array}$ & $\begin{array}{l}15 \\
25 \\
78 \\
68\end{array}$ & $\begin{array}{l}32 \\
42 \\
84 \\
81\end{array}$ & $\begin{array}{l}0.03 \\
0.13 \\
0.48 \\
0.26\end{array}$ & $\begin{array}{l}20 \\
27 \\
71 \\
82\end{array}$ & $\begin{array}{l}31 \\
41 \\
78 \\
78\end{array}$ \\
\hline
\end{tabular}

All scores are expressed as a percentage of the normal side. All are calculated in such a way that a higher score means better function.

SAP-sensory action potential

$\mathrm{p}$ - the probability value associated with a two-tailed $t$-test of differences of means in 1982 ; in 1985 there were no significant differences

The greater improvement in the microscope group in the interval between examinations is in keeping with the representation of the time-course of recovery by the curve of Figure 5. The $t$-tests carried out on the 1985 findings, which were considered to represent the final result, showed no significant differences between the two treatment groups. Moreover, some tests showed higher scores in the microscope group, some in the nomicroscope group.

However, before concluding that the use of the microscope had made no difference, it was necessary to take account of the differences between the groups in the patients' ages and the delay between injury and suture, which both exert strong influence on the degree of regeneration and recovery of function following suture of cut nerves (Onne 1962; Hallin, Wiesenfeld and Lungnegård 1981).

Age at suture had a profound effect, particularly on moving two-point discrimination, muscle power and the functional tests. It suggests there is particular deterioration after a delay of about two months.

Multivariate analysis was applied to establish whether controlling the age and delay revealed a consistent effect of the surgical technique. It did not. Whereas longer follow-up, lower age and shorter delay were consistently correlated with better results, use of the microscope per se was associated with small differences in the various test results, sometimes better, sometimes worse.

\section{DISCUSSION}

This study suggests that microsurgical epi-perineural suture holds no advantage over conventional epineural suture performed without the microscope. The series is small, but most of the correlations of measures of outcome with age at suture and follow-up time, and many with delay before suture, did reach significance at the 5\% level. More important, all these variables showed a 
consistent direction of effect; this was absent in the comparison between microscope and no-microscope groups.

It must be accepted that the conclusion derives from the experience of one surgeon. This has the advantage that any difference between groups could have been more safely ascribed to the surgical technique and equipment employed. However, the possibility that the operating microscope might enable another surgeon to produce better results cannot be excluded.

A further caveat is that the conclusion is reached on the basis of a particular set of measures of outcome. Despite the wide range of tests employed in this study, there can be no doubt that many aspects of nerve function, which ought to be studied, went unmeasured. This is not due to lack of effort on the authors' part, but to the fact that adequate tests do not yet exist. It is possible that there was a difference between the two groups, due to surgical technique and significant to the patients, yet invisible to the tests used.

The tests of spatial discrimination, both moving 2PD and the two wheel aesthesiometer tests, proved extremely difficult for both patient and examiner. Although no patient experienced any difficulty in performing them with the normal hand, only those who had their nerves sutured at the age of 13 or less were able

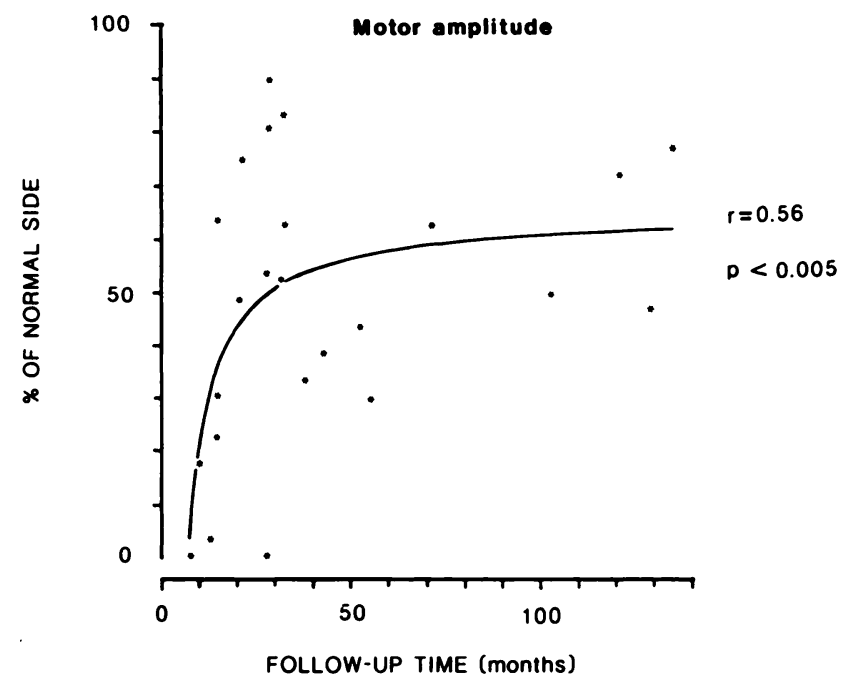

Fig. 5

Amplitude of muscle action potential as a function of time since suture. Results in 1982 with 24 cases.

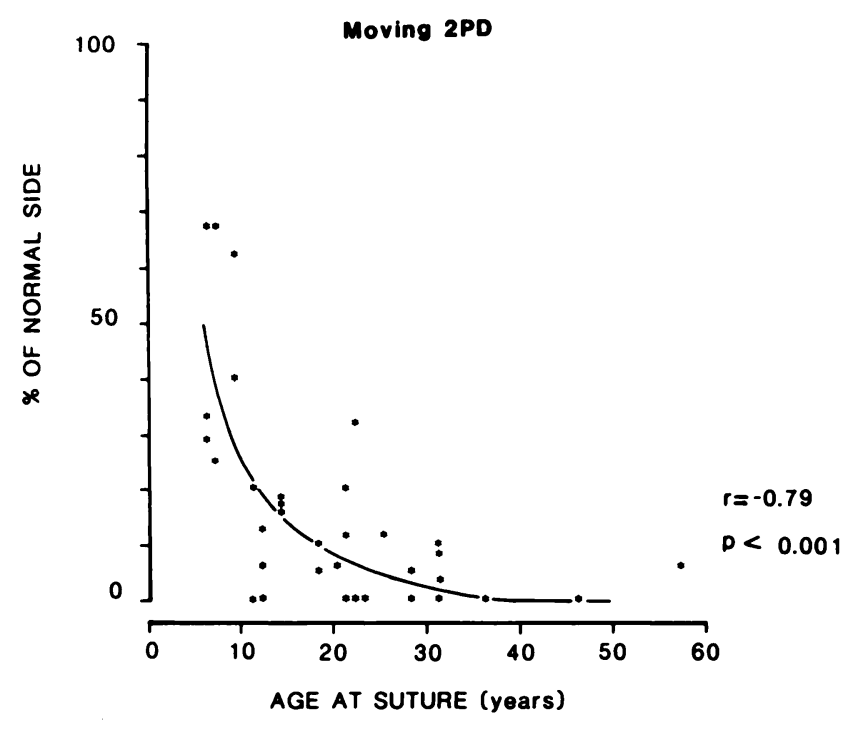

Fig. 6
Moving 2PD score as a function of age at suture. Combined 1982 and 1985 observations; some points represent two identical scores obtained in the same patient.

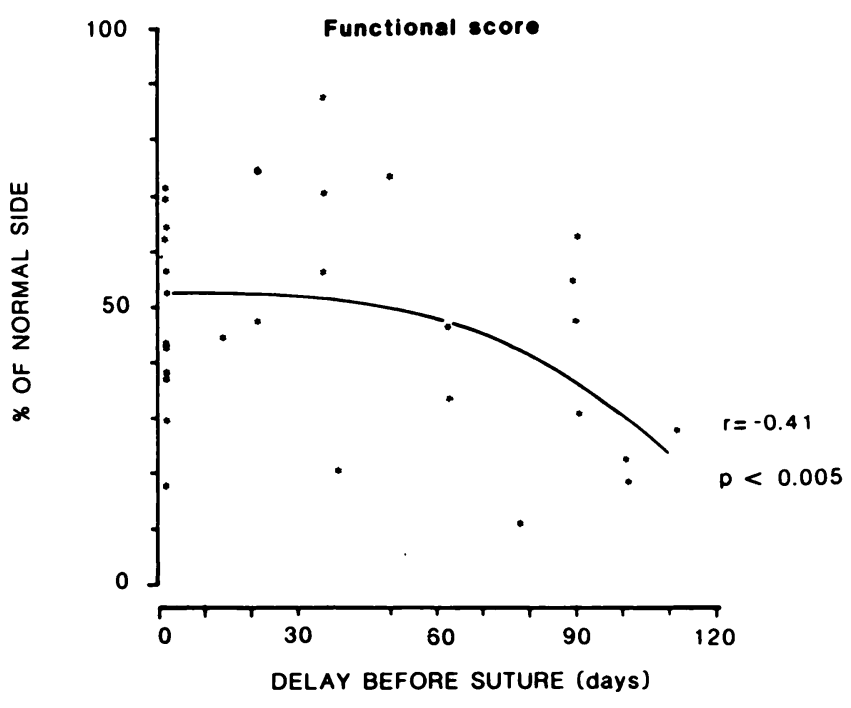

Fig. 7

Combined functional score as a function of delay before suture. All observations. 
to do so consistently with their injured hand. Applying strict criteria to establish threshold, and taking great care to avoid false positives due (for example) to asynchronous application of two points, most of the scores for older patients were at or near zero.

Paradoxically, many patients who could not distinguish two simultaneously applied points, even $30 \mathrm{~mm}$ apart, had no difficulty in correctly localising a single touch to within 5 to $10 \mathrm{~mm}$. This suggests that the neural mechanism for perceiving two points separated by a gap is complex, at least after nerve suture, and the idea that $2 \mathrm{PD}$ is a straight measure of the density of reinnervation may be misleading.

The influence of age and delay is always likely to be greater than the difference between treatments in trials of this kind and it is therefore imperative that they be effectively controlled. Ideally their effect should be neutralised by the use of large, properly randomised groups, but in practice this may not be possible.
Multivariate analysis is then the only way to avoid a stream of spurious findings.

Similarly, the time-course of recovery must be taken into account. The finding that function continues to improve for at least four years is in agreement with observations by previous authors (Nicholson and Seddon 1957; Onne 1962), though it is perhaps not widely appreciated by surgeons who treat these injuries. Comparisons between groups therefore require either minimum follow-up times of four years, or weighting of scores for each case by position on the recovery curve.

Grateful thanks are due to Mrs B. Smith, Occupational Therapist at the Queen's Medical Centre, Nottingham, who carried out all the timed tests of integrated hand function; Dr N. J. Smith, Consultant Clinical Neurophysiologist at the Queen's Medical Centre, Nottingham, who performed all the nerve conduction studies; and Mrs D. Hughes (illustrator) and Mr P. Starling (photographer), Physiological laboratory, University of Cambridge.

The latter part of this study was carried out when DM was in receipt of a Wellcome Foundation Research Fellowship and working in the Physiological Laboratory, University of Cambridge.

\section{REFERENCES}

Dellon AL. The moving two-point discrimination test: clinical evaluation of the quickly adapting fiber/receptor system. J Hand Surg 1978;3:474-81.

Donoso RS, Ballantyne JP, Hansen S. Regeneration of sutured human peripheral nerves: an electrophysiological study. J Neurol Neurosurg Psychiatry 1979;42:97-106.

Hallin RG, Wiesenfeld Z, Lungmegdrd $H$. Neurophysiological studies of peripheral nerve functions after neural regeneration following nerve suture in man. Int Rehabil Med 1981;3:187-92.

Hodes R, Larrabee MG, German WJ. Human electromyogram in response to nerve stimulation and conduction velocity of motor axons: studies on normal and on injured peripheral nerves. Arch Neurol Psychiat 1948;60:340-65.

Marsh DR. Use of a wheel aesthesiometer for testing sensibility in the hand: results in carpal tunnel syndrome. $J$ Hand Surg 1986;11-B:182-6.
Marsh D, Smith B. Timed functional tests to evaluate sensory recovery in sutured nerves. Br J Occupat Ther 1986;49:70-82.

Moberg E. Objective methods for determining the functional value of sensibility in the hand. J Bone Joint Surg [Br] 1958;40-B:454-76.

Nichobon OR, Seddon HJ. Nerve repair in civil practice: results of treatment of median and ulnar nerve lesions. Br Med J 1957;ii:1065-71.

Onne L. Recovery of sensibility and sudomotor activity in the hand after nerve suture. Acta Chir Scand 1962;Suppl 300:1-69.

Salvi V. Problems connected with the repair of nerve sections. Hand 1973;5:25-32.

Wy=m-Parry CB, Sutclifie B, Millar D. Rehabilitation of the hand. 2nd ed. London: Butterworths, 1966.

Young L, Wray RC, Weeks PM. A randomized prospective comparison of fascicular and epineural digital nerve repairs. Plast Reconstr Surg $1981 ; 68: 89-92$. 